\title{
UPPER BOUNDS FOR VERTEX DEGREES OF PLANAR 5-CHROMATIC GRAPHS $\left({ }^{1}\right)$
}

\author{
BY
}

\section{LEE W. JOHNSON}

\begin{abstract}
Upper bounds are given for the degrees of vertices in planar 5chromatic graphs. Some inequalities are derived for irreducible graphs which restrict the type of planar graphs that can be irreducible.
\end{abstract}

Introduction. Let $G$ be a planar graph with $n$ vertices $v_{1}, v_{2}, \cdots, v_{n}$, labelled so that $\rho\left(v_{i+1}\right) \geq \rho\left(v_{i}\right), i=1, \cdots, n-1$, where $\rho\left(v_{i}\right)$ is the degree of $v_{i}$. By a coloring of $G$ we shall mean a vertex coloring. It is natural to ask for sufficient conditions on $\rho\left(v_{n}\right)$ to guarantee that $G$ can be 4-colored. In [3], Malek and Skupień showed that $G$ can be 4-colored whenever $\rho\left(v_{n}\right) \geq n-6$. Their proof is based on the following observations:

$0-1$. Suppose $G$ is a planar graph with a vertex $v$ such that $\rho(v) \leq 4$. Let $G^{\prime}=G-v$ be the subgraph obtained by deleting $v$ and its incident edges from $G$. If $G^{\prime}$ can be 4-colored, then so can $G$ (cf., Berge [1]).

$0-2$. Let $k_{i}$ denote the number of vertices in $G$ of degree $i$. If $G$ has no vertices of degree 4 or less, then $k_{5} \geq 12+k_{7}+2 k_{8}+\cdots+(n-7) k_{n-1}$, with equality if and only if $G$ is maximal planar [3].

We include a brief sketch of their proof, since we use modifications of their technique later. From $0-2$, it follows that if $\rho\left(v_{n}\right) \geq n-6$, then $G$ has a vertex $v$ of degree 4 or less. Form $G^{\prime}=G-v$ and note that $\rho^{\prime}\left(v_{n}\right)$, the degree of $v_{n}$ in $G^{\prime}$, satisfies $\rho^{\prime}\left(v_{n}\right) \geq(n-1)-6$. Hence, $G^{\prime}$ has a vertex $v^{\prime}$ such that $\rho^{\prime}\left(v^{\prime}\right) \leq 4$. The vertex $v^{\prime}$ may be deleted from $G^{\prime}$ to form $G^{\prime \prime}$, and this process continued until a graph $H$ is obtained which is 4-colorable. Repeatedly using $0-1$, it follows that $G$ is 4 -colorable.

The result of Malek and Skupien can be improved somewhat without difficulty. Following Ore [4], an edge contraction in $G$ is defined by:

Received by the editors August 2, 1971.

AMS (MOS) subject classifications (1970). Primary 05C 15.

Key words and phrases. 4-color problem, irreducible graphs, vertex degree, planar graphs.

(1) This work was done while the author was an AEC Faculty Research Participant at the Savannah River Laboratory, Aiken, South Carolina, supported by AEC Contract AT(07-2)-1. 
Let $E=(u, v)$ be an edge in $G$. Form a graph $G^{\prime}$ from $G$ by identifying $u$ and $v$ and deleting loops and multiple edges.

A sequence of edge contractions is called a contraction (connected homomorphism). A graph $G$ is called contraction critical if any contraction $G^{\prime}$ of $G$ has a chromatic number less than the chromatic number $\chi^{(G)}$ of $G$. $G$ is called irreducible if $G$ is maximal planar, contraction critical and $\chi(G)=5$.

In Chapter 12 of [4], Ore gives numerous configurations which cannot occur in an irreducible graph $H$. In $\$ 2$, a result due to Errera [2] is used; namely, a minimal separating circuit $C$ in $H$ cannot consist of an even sequence of vertices of degree 5 and one arbitrary or two consecutive arbitrary vertices. In $\$ 2$, we use this to show $\rho\left(v_{n}\right) \geq n-8$ implies $G$ is 4 -colorable. However, these techniques rapidly become tedious. Therefore, in $\$ 3$ we confine ourselves to irreducible graphs, obtaining several inequalities and an upper bound on the largest vertex degree possible in an irreducible graph.

Upper bounds on the vertex degrees of a planar 5-chromatic graph. Let $G$ be a planar graph as before. For our purposes, we may as well assume $G$ is maximal, for if not we may add edges to $G$ and still retain the inequality $\rho\left(v_{n}\right) \geq$ $n-8$. Moreover, we may as well assume $G$ has no vertices of degree 4 or less. For if $G$ has a vertex $v$ of degree 4 or less, we may form $G_{1}=G-v$. The graph $G_{1}$ has $n-1$ vertices and the degree of $v_{n}$ in $G_{1}$ is no less than $(n-1)-8$. By repeatedly deleting vertices of degree 4 or less, we eventually obtain a graph $G_{k}$ that is either 4-colorable or has no vertices of degree 4 or less. In addition, if $G_{k}$ has $m$ vertices, the degree of $v_{n}$ in $G_{k}$ is no less than $m-8$.

Proposition 1. Let $\rho\left(v_{n}\right)=n-7$; then $G$ is 4 -colorable.

Proof. As $G$ is maximal and has no vertices of degree 4 or less, $k_{5}=$ $12+k_{7}+\cdots+(n-13) k_{n-7}$. Therefore, $k_{5}=n-1, k_{6}=k_{7}=\cdots=k_{n-8}=0$, $k_{n-7}=1$. The vertices adjacent to $v_{n}$ form a minimal separating circuit $C$, and as $C$ consists entirely of 5-vertices, $G$ cannot be irreducible. If $G$ is 4-colorable, we are through, so we may assume $\chi(G)=5$ and that $G$ is not contraction critical.

Hence, there is an edge in $G$, say $(u, v)$, that can be contracted so that the resulting graph $G^{\prime}$ is also 5-chromatic. Since all the vertices of $G$ except $v_{n}$ are 5-vertices, we may suppose $u$ is a 5-vertex. Figure 1(a) shows $u$ and its neighbors and Figure $1(\mathrm{~b})$ shows the contraction of the edge $(u, v)$, where $(b, v)$ and/or $(c, v)$ may have to be deleted in order that $G^{\prime}$ contain no multiple edges. 


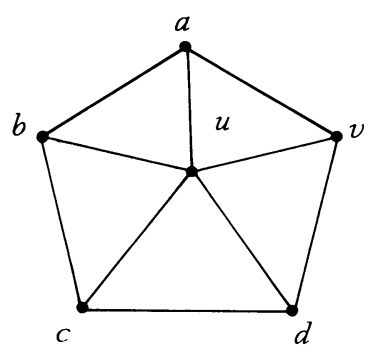

(a)

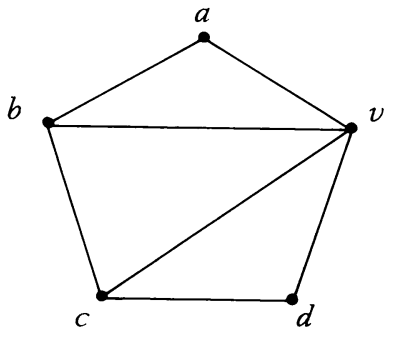

(b)

Figure 1

Add sufficient edges to $G^{\prime}$ to make the resulting graph maximal, and call the resulting graph $G_{0}$.

We claim $G_{0}$ has a vertex of degree 4. This is easily seen, for if $G$ does not contain edges from $b$ to $v$ or from $c$ to $v$, then $G^{\prime}=G_{0}$ and one of $a$ and $d$ is a 4-vertex. If $G$ contains an edge from $b$ to $v$ but no edge from $c$ to $v$, then $(b, v)$ is eliminated from Figure $1(\mathrm{~b})$ to form $G^{\prime}$. To get $G_{0}$, we add the edge $(a, c)$ to $G^{\prime}$, and now one of $b$ and $d$ must be a 4-vertex. Similarly, if $G$ has edges from $b$ to $v$ and $c$ to $v$, then edges $(a, c)$ and $(a, d)$ must be added to $G^{\prime}$ to get $G_{0}$, forcing one of $b$ and $v$ to be a 4-vertex.

Thus, $G_{0}$ has $n-1$ vertices, is maximal planar, has a vertex of degree 4 and has only one vertex of degree larger than 5. Further, each vertex of $G_{0}$ has degree 3 or larger. Now form a sequence of maximal planar graphs $G_{0}, G_{1}, \ldots, G_{k}$ as follows: if $r$ is a vertex of $G_{0}$ of degree 4 or less, delete $r$ from $G_{0}$ and add sufficient edges so that the result $G_{1}$ is maximal planar. If the degree of $r$ is 3 , no edges need be added while if the degree of $r$ is 4 only one edge can be added. Clearly $G_{1}$ has a vertex of degree 4 or less, has $n-2$ vertices only one of which has degree larger than 5 and is maximal. We obtain $G_{2}$ from $G_{1}$ in the same fashion that $G_{1}$ was obtained from $G_{0}$, terminating the sequence when one of the graphs $G_{k}$ is 4-colorable.

Since $G_{k}$ is 4-colorable, so is $G_{0}$. But if $G_{0}$ is 4-colorable, then so is $G^{\prime}$ which is a contradiction. Hence, $G$ must have been 4 -colorable.

We only sketch the proof of Proposition 2 as it is similar to the proof of Proposition 1.

Proposition 2. Let $\rho\left(v_{n}\right)=n-8$, then $G$ is 4-colorable.

Proof. Since $G$ is maximal and has no vertices of degree 4 or less, $k_{5}=$ $12+k_{7}+\cdots+(n-14) k_{n-8}$. Thus, $k_{5}=n-2, k_{6}=1, k_{7}=k_{8}=\cdots=k_{n-9}=0$, $k_{n-8}=1$. As before, $G$ cannot be irreducible, and hence $G$ is either 4-colorable, or not contraction critical. 
If $G$ is not contraction critical, we suppose the edge $(u, v)$ can be contracted to form $G^{\prime}$, where $\chi\left(G^{\prime}\right) \geq \chi(G)$. Add sufficient edges to $G^{\prime}$ to form $G_{0}$ which is maximal. Recall that, in $G, \rho\left(v_{n-1}\right)=6$ and note that if $\rho(u)=5$ and $v=v_{n}$ or $v=v_{n-1}$, then the argument in Proposition 1 shows $G_{0}$ has a vertex of degree 4. If $\rho(u)=6$ and $v=v_{n}$, then a slight modification of the argument in Proposition 1 shows $G_{0}$ has a vertex of degree 4 , i.e., the pentagons in Figure 1 are now hexagons. The only troublesome case is when $\rho(u)=\rho(v)=5$. We refer to Figure 1 to handle this case.

If $G$ does not contain the edges $(b, v)$ and $(c, v)$, then $G^{\prime}=G_{0}$. If one of $a$ or $d$ had degree 5 in $G$, then it has degree 4 in $G_{0}$. Thus, suppose that $a=v_{n}$ and $d=v_{n-1}$. In $G_{0}, v_{n}$ has degree $(n-1)-8, d$ has degree 5 and $v$ has degree 6 . Therefore, since $G_{0}$ has $n-1$ vertices, one of which is of degree $(n-1)-8$, we may apply the same reasoning to $G_{0}$ as we did to $G$ and conclude $G_{0}$ is not contraction critical. Similar arguments apply if $G$ contains one or both of the edges $(b, v)$ and $(c, v)$ and show that $G_{0}$ either has a vertex of degree 4 or is not contraction critical.

If $G_{0}$ is not contraction critical, contract an edge of $G_{0}$ to form $G_{0}^{\prime}$, where $\chi\left(G_{0}^{\prime}\right) \geq \chi\left(G_{0}\right)$. Add sufficient edges to $G_{0}^{\prime}$ to form $G_{1}$ which is maximal, and note $\chi\left(G_{1}\right) \geq \chi(G)$. Continue until a graph $G_{k}$ is formed, where $G_{k}$ is 4-colorable or $G_{k}$ has a vertex of degree 4 or less. Note that in each $G_{i}, 0 \leq i \leq k$, if $G_{i}$ has $m$ vertices then $G_{i}$ has one vertex of degree $m-8$, one of degree 6 and $m-2$ vertices of degree 5. Thus, we may as well assume $G_{0}$ has a vertex of degree 4 , say $r$.

We now form a sequence of maximal planar graphs $G_{i}$, as in Proposition 1, by repeatedly deleting vertices of degree 4 or less. The only difficulty occurs when a vertex $r_{i}$ of degree 4 in $G_{i}$ is adjacent to two vertices of degree 6 or more, as in Figure 2.

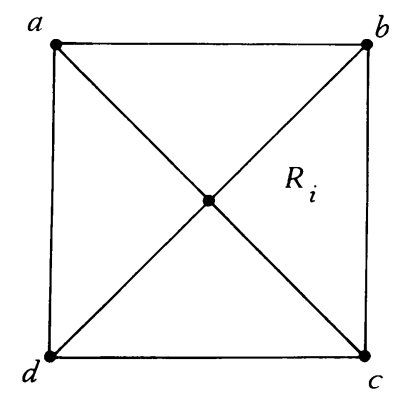

Figure 2

If the degrees of $a$ and $c$ are larger than 5 and if the edge $(a, c)$ is in $G_{i}$, then $G_{i+1}$ is formed by deleting $r_{i}$ and adding the edge $(b, d)$. If $G_{i+1}$ has no 
vertices of degree 4 or less, then $b$ and $d$ must have had degree 5 in $G_{i}$ and hence have degree 5 in $G_{i+1}$. In $G_{i}$, one of $a$ and $c$ must have had degree 6 , for the process of forming the sequence does not increase the degrees of vertices. Suppose $a$ had degree 6 in $G_{i}$, then $a$ has degree 5 in $G_{i+1}$. Further, all the vertices of $G_{i+1}$, except $a$, have degree 5. Suppose $G_{i+1}$ has $m$ vertices. Then as $G_{i+1}$ is maximal and has no vertices of degree 4 or less and $m-1$ vertices of degree 5 , we may use $0-2$ to obtain $m-1=12+k_{7}+\cdots+(m-7) k_{m-1}$, with $k_{7}+k_{8}+\cdots+k_{m-1}=1$. Thus, $k_{m-7}=1$ so Proposition 1 applies, and $G_{i+1}$ is 4-colorable. As before, whenever one of the sequence $G_{j}$ is 4-colorable, so is $G$.

Bounds and inequalities for irreducible graphs. In this section, we derive some inequalities which restrict the types of planar graphs which can be irreducible. The interest in irreducible graphs is, of course, engendered by the fact that a planar 5-chromatic graph must be contractible to an irreducible graph.

Hence, the 4 -color conjecture is equivalent to showing there can be no irreducible graphs.

Let $H$ be an irreducible graph with $n$ vertices. In Ore [4], are found configurations which cannot occur in $H$. First, if $v$ is a vertex of $H$, then $\rho(v) \geq 5$. Following Ore, if $\rho(v)=5$ or $\rho(v)=6$ then $v$ is called a minor vertex, otherwise $v$ is called a major vertex. If $\rho(v)=k$, then $v$ is called a $k$-vertex. If $H$ is to be irreducible, the following must hold:

(a) Each 5-vertex has a major neighbor.

(b) Each 5-vertex with exactly 1 major neighbor has at least 2 neighbors which are 6-vertices.

(c) Each 6-vertex has a major neighbor.

(d) Each 6-vertex with exactly 1 major neighbor has no more than two 5vertices as neighbors.

(e) Each 7-vertex with exactly 1 major neighbor has no more than four 5vertices as neighbors.

(f) Each 7-vertex with no major neighbor has no more than three 5-vertices as neighbors.

(g) Each $k$-vertex, $k \geq 8$, has no more than $k-2$ neighbors of degree 5 .

Condition $(\mathrm{g})$ is due to Errera, the others are results of Birkhoff, Franklin and Winn.

We write $k_{7}=R+S+T, k_{6}=M+N$ and $k_{5}=A+B$, where

$R=$ number of 7 -vertices with 2 or more major neighbors;

$S=$ number of 7 -vertices with exactly 1 major neighbor;

$T=k_{5}-R-S$;

$M=$ number of 6 -vertices with 2 or more major neighbors; 
$N=k_{6}-M$

$A=$ number of 5-vertices with 2 or more major neighbors;

$B=k_{5}-A$.

Let $\lambda$ denote the maximal degree of the vertices of $H$. Clearly, $H$ must contain at least $2 A+B$ major vertices. Using (g), together with (e) and (f), we obtain the inequality

$$
2 A+B \leq 5 R+4 S+3 T+6 k_{8}+7 k_{9}+\cdots+(\lambda-2) k_{\lambda}
$$

or

$$
2 A+B+5+2 T \leq 5 k_{7}+6 k_{8}+\cdots+(\lambda-2) k_{\lambda} .
$$

From (b) and (d)

$$
2 B \leq 4 M+2 N
$$

or

$$
B+N \leq 2 k_{6}
$$

Finally,

$$
2 A+B+2 R+S+2 M+N \leq 7 k_{7}+8 k_{8}+\cdots+\lambda k_{\lambda}
$$

Adding (2), (5) and twice (4),

$$
4 k_{5}+2 k_{6}+2 k_{7}+N \leq 4 k_{6}+12 k_{7}+\cdots+(2 \lambda-2) k_{\lambda}
$$

so if we add $2 n+8 k_{5}+6 k_{6}$ to both sides of (6) and use $6 n-12=5 k_{5}+6 k_{6}+$ $\cdots+\lambda k_{\lambda}$, we obtain

$$
12 k_{5}+8 k_{6}+2 k_{7}+N \leq 10 n-24 .
$$

In (7), write $12 k_{5}+8 k_{6}=7\left(k_{5}+k_{6}\right)+5 k_{5}+k_{6}$ and note that $\left(k_{5}+k_{6}\right)=n-$ $k_{7}-k_{8}-\cdots-k_{\lambda}$, while $k_{5}=12+k_{7}+2 k_{8}+\cdots+(\lambda-6) k_{\lambda}$. Thus, (7) is transformed to

$$
k_{6}+3 k_{8}+8 k_{9}+\cdots+(5 \lambda-37) k_{\lambda}+N \leq 3 n-84 .
$$

Another formulation of (7) is gotten by setting $n=k_{5}+k_{6}+\cdots+k_{\lambda}$ and using $2 k_{5}=2\left(12+k_{7}+\cdots+(\lambda-6) k_{\lambda}\right)$ to obtain

(9) $24+k_{12}+2 k_{13}+\cdots+(\lambda-11) k_{\lambda}+N / 2 \leq k_{6}+3 k_{7}+3 k_{8}+2 k_{9}+k_{10}$.

While inequalities (7), (8) and (9) are all equivalent, each finds its own use in determining what an irreducible graph must look like, as will be demonstrated below.

Inequality (8) gives an upper bound for the maximal degree that a vertex in $H$ can have, namely

$$
\lambda \leq(3 n-47) / 5 \text {. }
$$


It has been shown recently [5] that a graph with 39 or fewer vertices is 4colorable, but it is unknown whether a graph with 40 vertices is 4 -colorable. When $n=40$ in (10), we obtain $\lambda \leq 14$. The use of inequalities (1)-(9) will determine the possible degrees of vertices in an irreducible graph with 40 vertices and a vertex of degree 14 .

Statement (8) gives $k_{6}+3 k_{8}+\cdots+33 k_{14} \leq 36$ and, hence, $0 \leq k_{6} \leq 3,0 \leq$ $k_{8} \leq 1, k_{9}=k_{10}=\cdots=k_{13}=0$, and $k_{6}+3 k_{8} \leq 3$. Statement (9) gives $27 \leq$ $k_{6}+3 k_{7}+3 k_{8}$, or $k_{7} \geq 8$. By statement (7), $6 k_{5}+4 k_{6}+k_{7} \leq 188$. Using $k_{5}=$ $12+k_{7}+2 k_{8}+8 k_{14}$, we obtain $7 k_{7}+4 k_{6}+12 k_{8} \leq 68$. We have deduced above, $4 k_{6}+12 k_{8} \leq 12$, thus $k_{7}=8$.

From $k_{5}=12+k_{7}+\cdots+(\lambda-6) k_{\lambda}$, it is easy to deduce $n=12+k_{6}+$ $2 k_{7}+\cdots+(\lambda-5) k_{\lambda}$. Thus, $\lambda=14, k_{7}=8$ implies $3=k_{6}+3 k_{8}$. If $k_{8}=1$ then $k_{6}=0$ and so $B=0$. Hence, $k_{5}=30=A$, and in (2) we obtain $60+5+2 T \leq$ $40+6+12$, an impossibility. Hence, $k_{5}=28, k_{6}=3, k_{7}=8, k_{8}=k_{9}=\cdots=$ $k_{13}=0, k_{14}=1$.

More information can be gained from (2), (4) and (5). It is not hard to show the only possibilities are $A=22, B=6, M=3, N=0$ and $R=7, S=0, T=1$ or $R=6, S=7, T=0$.

\section{REFERENCES}

1. C. Berge, The theory of graphs and its applications, Methuen, London; Wiley, New York, 1962. MR 24 \#A2381.

2. A. Errera, Une contribution au problème des quatres couleurs, Bull. Soc. Math. France 53 (1925), 42-55.

3. M. Malek and Z. Skupien, On the maximal planar graphs and the four colour problem, Prace Mat. 12 (1969), 205-209. MR 39 \#5418.

4. O. Ore, The four color problem, Pure and Appl. Math., vol. 27, Academic Press, New York, 1967. MR $36 \# 7$.

5. O. Ore and G. Stemple, Numerical methods in the four color problem, Recent Progress in Combinatorics (Proc. Third Waterloo Conf. on Combinatorics, 1968), Academic Press, New York, 1969.

SAVANNAH RIVER LABORATORY, E. I. DU PONT DE NEMOURS \& CO., AIKEN, SOUTH CAROLINA 29801

Current address: Department of Mathematics, Virginia Polytechnic Institute and State University, Blacksburg, Virginia 24601 